\title{
A dual-probe recognition memory task for use during sustained operations
}

\author{
DAVID F. DINGES and WAYNE G. WHITEHOUSE \\ The Institute of Pennsylvania Hospital and University of Pennsylvania, Philadelphia, Pennsylvania
}

\begin{abstract}
Although memory has been shown to be greatly affected by sleep loss, there is an absence of data on the effect of sustained work on recognition memory. This is unfortunate because recognition, unlike free recall, can be analyzed to separate effects on memory sensitivity from effects on report bias. Based upon work with single-probe recognition memory tasks, we developed a dual-probe recognition task for practical use in sustained work paradigms. The task requires $20 \mathrm{~min}$ to complete and yields an array of performance variables that can be used to conduct signal detection analyses, to assess serial-position effects, and to evaluate speed-accuracy functions.
\end{abstract}

Performance on memory tasks during sleep deprivation, with and without sustained work demands, has been studied very little compared with vigilance and other kinds of cognitive performance, despite the fact that short-term memory impairment following a night without sleep is among the earliest performance effects documented (see Williams, Lubin, \& Goodnow, 1959). Furthermore, as Johnson (1982) has noted, the lapse hypothesis (Williams et al., 1959), which has been invoked to explain the major effects of sleep loss on human performance, cannot readily account for certain effects of sleep loss on human memory (Williams, Gieseking, \& Lubin, 1966).

The most extensive investigations of information retention as a function of sleep deprivation have been carried out by Elkin and Murray (1974) and by Polzella (1974, 1975,1978 ), using assessments of recognition memory. Working independently, these investigators developed different types of single-probe recognition memory tasks and studied the performance of humans, with and without sleep loss, on those tasks. Their efforts converged to document the impairment of recognition performance following a single night without sleep; in each case, the impairment appeared to involve decrements in attention to and rehearsal of the critical stimuli.

The study of recognition memory during sustained operations, when sleep loss is accompanied by quasicontinuous workloads, has not been reported, although memory-search and free-recall performances have been assessed (e.g., Naitoh, 1981). Recognition testing,

This research was supported in part by Office of Naval Research Contract NO0014-80-C-0380 to the first author and in part by a grant from the Institute for Experimental Psychiatry. We thank Donald J. Polzella for details concerning his development of a visual probe recognition task, and D. J. Murray for details of his auditory probe recognition memory task, both of which formed the basis for the development of our task. We acknowledge the helpful suggestions of Stephen R. Fairbrother, Emily Carota Orne, Martin T. Orne, David A. Soskis. John W. Powell, Ann Maliniak Whitehouse, and Mary F. Auxier. Reprint requests should be sent to D. F. Dinges. Unit for Experimental Psychiatry, 111 N. 49th Street, Philadelphia, PA 19139-2798. however, offers a decisive and conceptually significant advantage with such a regimen, in that it permits an empirical separation of memory and decision processes through the application of procedures derived from signal detection theory (see Murdock, 1982). ${ }^{1}$

In studying sustained, quasi-continuous work demands accompanied by sleep loss, we sought to measure recognition memory with a relatively brief, practical task. The pioneering works of Elkin and Murray (1974) and of Polzella (1974) served as the basis for our development of a dual-probe recognition memory task. This task combines certain key features of the tasks developed by these earlier investigators with a dual-probe procedure, to yield the maximum amount of data in a 20-min trial. In this paper we describe this task and the variety of performance variables it produces for analysis.

\section{METHOD}

\section{The Single-Probe Recognition Paradigm}

A probe recognition memory task typically involves presentation of a stimulus series (of variable number) followed by a single-probe stimulus. The retention interval between the final stimulus in the series and presentation of the probe can range from $0 \mathrm{sec}$ (i.e., immediate) to varying delays. Stimulus presentation typically occurs in either the auditory modality or the visual modality. Some tasks require the subject to repeat the stimuli as they are presented to ensure sensory registration.

The subject's task is to decide whether or not the probe stimulus was one of the stimuli encountered in the preceding series. Following this decision, the subject is usually required to give a confidence estimate on a predetermined (i.e., n-point rating) scale, with regard to the accuracy of his/her yes-no decision. The latency or reaction time (RT) to make the yes-no decision concerning the probe stimulus may also be recorded along with the decision and confidence rating.

The advantages of the single-probe recognition paradigm are that: (1) the probe can correspond to any 
item position in the stimulus series, thereby permitting as assessment of retroactive and proactive inhibition effects (e.g., Polzella, 1974); (2) the a priori probability that the probe will be a repeat of one of the stimulus items can be fixed at .50 or at any other probability level; (3) one can calculate the proportion of hits, false alarms, correct rejections, and misses; (4) the proportions of hits and false alarms can be used in signal detection analyses, which can empirically dissociate memory sensitivity $\left(d^{\prime}\right)$ and response bias $(\beta)$; and (5) memory operating characteristic (MOC) curves can be evaluated by combining the decision and confidence data. In short, the single-probe recognition paradigm permits a different and more extensive assessment of the nature and time course of memory alterations than does a recall performance paradigm.

\section{A Dual-Probe Recognition Task}

Adaptation of the probe recognition paradigm to the study of effects of sustained operations on recognition memory required that it be structured to yield the maximum amount of information in as brief a period of time as possible. In the work of both Polzella (1974) and Elkin and Murray (1974), performance trials lasted 30 to $60 \mathrm{~min}$. We felt that a more practical task for sustained operations should be shorter without compromising the overall utility and sensitivity of the single-probe paradigm. To accomplish this, we adapted the procedure so that it retained characteristics of the tasks developed by earlier investigators, but included two probes rather than one per stimulus series.

Stimuli and probes. A stimulus series for one trial of the dual-probe task begins with six number-triplets (e.g., 369,742 , etc.), presented through a loudspeaker or headphones at the rate of one stimulus every $2.5 \mathrm{sec}$. This task is similar to the task developed by Elkin and Murray (1974). The number triplets were extracted from a table of random numbers. Each set of six number triplets was followed by $15 \mathrm{sec}$ of silence, which terminated in a 1$\mathrm{kHz}, 2$-sec tone followed by the first probe triplet. The subject responded either "yes" or "no" to this first probe. Another $15 \mathrm{sec}$ of silence followed and terminated in a second tone, which was followed immediately by the second probe triplet, to which the subject again responded either "yes" or "no." After the second probe, an interval of $25 \mathrm{sec}$ of silence elapsed before a new set of six triplets was begun. ${ }^{2}$

All stimuli and probes were prerecorded on audiotape by the first author. A 20 -min trial consisted of 18 sets of six number triplets, with each set followed by two probe stimuli. Subjects were instructed to repeat each number triplet and probe stimulus aloud as they heard it, to ensure that all stimuli were perceived. ${ }^{3}$

Decision, latency, and confidence. Subjects were instructed to decide as accurately and rapidly as possible whether a probe item was or was not one of the six triplets presented. The response consisted of pressing one of two levers (marked "yes" and "no") and simultaneously say- ing "yes" or "no" aloud as the selected lever was pressed. The lever response illuminated one of two bulbs, visible only to the experimenter. This redundancy of decision response (i.e., lever and oral responding) was added to measure discrepancies in response modes; it is not an essential aspect of the task.

Response latency was defined as the time between the onset of the tone signaling the probe, and the time at which the subject pressed a decision lever; Polzella (1974) incorporated this measure, but Elkin and Murray (1974) did not. Subjects' RTs were measured in the dual-probe task by having the experimenter manually start an electronic timing device that accumulated time in milliseconds until the subject closed a circuit by pressing a decision lever. The response latency was then recorded by the experimenter and the timer was reset manually.

Immediately after each probe decision response, the subject indicated confidence in the accuracy of the response by assigning a value from a 4-point confidence scale, where 0 indicated "not confident," 1 "a little confident," 2 indicated "fairly confident," and 3 corresponded to "very confident."

Performance characteristics. The task was structured so that the a priori probability of any given probe being a member of the prior stimulus set was .50. Thus, chance performance necessarily would average to $50 \%$. Our experience with the task indicates that healthy young adults, who are not sleep-deprived or otherwise stressed, consistently perform between $50 \%$ and $100 \%(M=70 \%)$ on a 20 -min trial. There is little indication of a practice effect on the task, although a minimum of two baseline trials, separated by a day or more, is recommended.

Within each of the 20-min stimulus trials, half of the 18 probes in the first nine stimulus sets (i.e., first $10 \mathrm{~min}$ of a trial), and half in the second nine stimulus sets (i.e., last $10 \mathrm{~min}$ of a trial) were part of the stimulus series, so that a correct decision was "yes." Thus, the .50 a priori probability of a hit (and correct rejection) characterized each half of a 20-min trial. Similarly, half of the probes presented first and half of the probes presented second were part of the stimulus series; that is, a .50 a priori probability existed separately for first probes and for second probes within each half of a task trial.

Those probe items that were part of the prior six-triplet stimulus set were equiprobable within a trial with respect to each of the six serial positions (i.e., $1 / 6$ or .167). Similarly, each serial position was represented by probes either once or twice in each 10-min half of a trial, with the proviso that each position was represented a total of three times in the 20 -min trial (i.e., $3 / 18=1 / 6=.167$ ).

Performance measures. The task can yield a variety of performance measures, depending upon the purpose of the analysis. In addition to measuring total trial performance, the structure of the task makes it possible to conduct analyses on performance from each half of a trial, in order to assess changes as a function of time on the task.

Hits, false alarms, correct rejections, and misses can be calculated independently of confidence ratings, or in 
conjunction with confidence ratings for the purpose of generating MOC curves and for calculating measures of memory sensitivity $\left(\mathrm{d}^{\prime}\right)$ and response bias $(\beta)$. Other signal detection theory measures of memory processes are possible (cf. Polzella, 1974), as are assessments of serialposition effects on the task. Response latencies can be used to derive speed-accuracy functions, which are often negatively correlated during sustained operations. In addition, the dual-probe nature of the task permits a comparison of performance on the first probe (i.e., 15-sec delay) with performance on the second probe (i.e., 30-sec delay), using many of the measures mentioned above.

\section{DISCUSSION}

It remains to be seen to what extent the various performance measures available from the dual-probe recognition memory task change as a function of combined sleep loss, circadian oscillation, and quasi-continuous work demands. A study is currently being completed to begin providing answers to these questions. It is hoped that future investigations of sustained operations will consider the practical advantages of the dual-probe recognition memory task as a supplement to human performance test batteries.

\section{REFERENCES}

Elkin, A. J., \& MUrRay, D. J. (1974). The effects of sleep loss on short-term recognition memory. Canadian Journal of Psychology, 28, 192-198.

Johnson, L. C. (1982). Sleep deprivation and performance. In W. B. Webb (Ed.), Biological rhythms, sleep, and performance (pp. 111 141). Chichester, England: Wiley.

Murdock, B. B. (1982). Recognition memory. In C. R. Puff (Ed.), Handbook of research methods in human memory and cognition (pp. 1 26). New York: Academic Press.

NaITOH, P. (1981). Circadian cycles and restorative power of naps.
In L. C. Johnson, D. I. Tepas, W. P. Colquhoun, \& M. J. Colligan (Eds.), Biological rhythms, sleep and shiftwork (pp. 553-580). New York: Spectrum.

NaIToH, P. (1983). Editorial: Signal detection theory as applied to vigilance performance of sleep-deprived subjects. Sleep, 6, 359-361.

Polzella, D. J. (1974). The effect of sleep deprivation on short-lerm memory (Technical Report No. 47). Ann Arbor, MI: University of Michigan, Human Performance Center.

Polzella, D. J. (1975). The effects of sleep deprivation on short-term recognition memory. Journal of Experimental Psychology, 104, 194-200.

Polzella, D. J. (1978). Effect of sleep deprivation on response threshold for signal detectability parameter, $\beta$. Perceptual \& Motor Skills, 47, 1189-1190.

Williams, H. L., Gieseking, C. F., \& Lubin, A. (1966). Some effects of sleep loss on memory. Perceptual \& Motor Skills, 23, 1287-1293.

Williams, H. L., Lubin, A., \& Goodnow, J. J. (1959). Impaired performance with acute sleep loss. Psychological Monograhs, 73(14, Whole No. 484).

\section{NOTES}

1. Although there is some controversy over whether signal detection theory can be appropriately applied to vigilance performance of sleep-deprived subjects (cf. Naitoh, 1983), there is a considerable scientific literature on, and theoretical justification for, its application to recognition memory performance.

2. We originally used nine stimuli per trial and 20 trials per set, but discovered that this task was too difficult. The use of a 15-sec delay prior to the first probe was chosen because Elkin and Murray (1974) demonstrated that a delayed probe (but not a probe immediately following the stimulus set) yielded performance that was adversely affected by $55 \mathrm{~h}$ of sleep loss; Polzella (1974) used only no-delay probes.

3. Four 20-min stimulus trials (each trial consists of 18 stimulus sets and 36 probes) are available on $1 / 4$-in. audiotape on a 7 -in. reel, or on paper that also shows the probes and whether each was or was not one of the six stimuli in a series.

4. Future modifications of the dual-probe task involve microcomputer automation of task components.

5. Polzella (1974) used a 3-point scale, and Elkin and Murray (1974) used a 6-point scale. We have found that a 4-point scale is easily remembered and used by even severely sleep-deprived subjects. It also avoids the problems associated with middle responses on odd-numbered scales. 